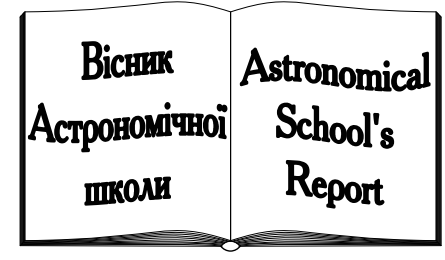

ISSN 1607-2855

УДК 524.3

\title{
Эволюция нуклидов
}

\section{Н.С. Комаров}

НИИ “Астрономическая обсерватория” Одесского национального университета им. И.И.Мечникова

В кратком обзоре обсуждены современные проблемы дальнейшей эволючии нуклидов после образования самых легких из них H, D, ${ }^{3} \mathrm{He},{ }^{4} \mathrm{He}$ и, возможно, ${ }^{7}$ Li через 100 секунд после Большого Взрыва. Рассмотрены возможные пути эволючии нуклидов в результате: равновесного прочесса одновременного синтеза; неравновесного процесса захвата нейтронов; термоядерного синтеза. Приведена «стандартная» кривая распространенности химических элементов, как результат эволюиии нуклидов от Большого Взрыва до настоящего времени. Обсуждены результаты определения содержания химических элементов в атмосферах звезд разных спектральных типов и рассмотрены механизмы обогащения межзвездной и межгалактической среды тяжелыми элементами. Особое внимание уделено определению содержания изотопов, как наиболее важных тестов того или иного процесса термоядерного синтеза. Проведено сравнение современных данных о содержании нуклидов в метеоритах с данными, полученными для атмосфер звезд, и оно показало на значительные различия, которые не могут быть объяснены в рамках современной теории термоядерного синтеза.

ЕВОЛЮЦІЯ НУКЛІДІВ, Комаров М.С. - У короткому огляді обговорені сучасні проблеми подальшої еволюиї̈ нуклідів після утворення самих легких з них $\mathrm{H}, \mathrm{D},{ }^{3} \mathrm{He},{ }^{4} \mathrm{He} i$, можливо, ${ }^{7} \mathrm{Li}$ через 100 секунд після Великого Вибуху. Розглянуто можливі шляхи еволюиії нуклідів у результаті: рівноважного прочесу одночасного синтезу; нерівновагого процесу захоплення нейтронів; термоядерного синтезу. Приведена “стандартна" крива поширеності хімічних елементів, як результат еволючії нуклідів від Великого Вибуху дотепер. Обговорено результати визначення вмісту хімічних елементів в атмосферах зірок різних спектральних типів і розглянуті механізми збагачення міжзоряного $i$ міжгалактичного середовища важкими елементами. Особлива увага приділена визначенню вмісту ізотопів, як найбільш важливих тестів того чи іншого процесу термоядерного синтезу. Проведено порівняння сучасних даних про вміст нуклідів у метеоритах з даними, отриманими для атмосфер зірок, і воно показало на значні розходження, що не можуть бути пояснені в рамках сучасної теорії термоядерного синтезу.

THE EVOLUTION OF NUCLIDES, by Komarov N.S. - In the brief review the modern problems of the further evolution nuclides after formation of easiest of them $\mathrm{H}, \mathrm{D},{ }^{3} \mathrm{He},{ }^{4} \mathrm{He}$ and, probably, ${ }^{7} \mathrm{Li}$ in 100 seconds after the Big Bang are discussed. The possible ways of evolution nuclides in result are considered: equilibrium process of simultaneous synthesis; nonequilibrium process of capture of neutrons; nuclear of synthesis. The "standard" curve of distribution of chemical elements, as result of evolution nuclides from the Big Bang till now is given. The results of determination of the contents of chemical elements in atmospheres of stars of different spectral types are discussed and the mechanisms of enrichment of interstellar and intergalactic environment by heavy elements are considered. The special attention is given to determination of the contents of isotopes, as most important tests of this or that process nucleosynthesis. The comparison of the modern data about the contents of nuclides in meteorites with the data received for atmospheres of stars is carried out and it has shown on significant distinctions, which can not be explained within the framework of the modern theory of nucleosynthesis.

\section{ВВЕДЕНИЕ}

В данном обзоре мы остановимся на проблеме эволюции нуклидов. Сначала хотелось бы остановиться на терминологии. Когда определяются содержания химических элементов и их изотопов, мы имеем дело с атомными и молекулярными линиями поглощения или эмиссии химических элементов и ионов, а также с их соединениями, а, именно, с их атомными или молекулярными спектральными харак- 
теристиками. Но, когда мы имеем дело с процессом образования новых более тяжелых химических элементов, то это можно считать одной из эволюционных стадий более легких нуклидов. Эта эволюция проходит в результате тех или иных ядерных процессов, то логичнее говорить об эволюции нуклидов (ядер химических элементов). Более того, самые легкие нуклиды H, D, ${ }^{3} \mathrm{He},{ }^{4} \mathrm{He},{ }^{7} \mathrm{Li}$ образовались примерно через 100 с после Большого Взрыва. Образование первых химических элементов, когда у легких ядер появились электронные оболочки, произошло на $3 \cdot 10^{5}$ лет позднее нуклидов, по крайней мере, водорода, дейтерия и гелия.

\section{РОЖДЕНИЕ ПЕРВЫХ НУКЛИДОВ}

Наш будущий материальный мир спасло то, что число частиц несколько превышало число античастиц и аннигиляция не могла быть полной. Этот небольшой излишек «выживших» барионов и есть вся барионная материя сегодняшней Вселенной. Родившиеся в результате Большого Взрыва фотоны к настоящему времени остыли до температуры $2,7 \mathrm{~K}$ и присутствуют во Вселенной в виде реликтового излучения, впервые зарегистрированного в 1964 г. Из сравнения их числа с количеством барионов в современной Вселенной следует, что после аннигиляции осталась только $10^{-9}$ часть. Примерно через $1 \mathrm{c}$ после Большого Взрыва $\left(T=10^{10} \mathrm{~K}\right.$, размер Вселенной увеличился до $10^{14}$ км, или 10 св.л.) плотность частиц снизилась до такого значения $\left(\sim 10^{5}\right.$ г $\left.\mathrm{cm}^{3}\right)$, при котором взаимодействия с участием нейтрино становятся настолько редкими, что они не могут больше находиться в термодинамическом равновесии с другими частицами. Эти нейтрино начинают жить своей независимой жизнью, свободно двигаясь по Вселенной (нейтринное реликтовое излучение). Если нейтрино имеет нулевую массу покоя, то такое излучение должно иметь температуру всего 2 К, а при ненулевой массе нейтрино, скажем порядка 10 эВ $\left(\sim 2 \cdot 10^{-33} \Gamma\right)$, их температура будет выше абсолютного нуля всего на несколько тысячных градуса. По этой причине, а также из-за очень малой вероятности взаимодействия нейтрино с веществом, нейтринное реликтовое излучение до сих пор не зарегистрировано.

По прошествии нескольких секунд, при энергиях ниже $10^{6}$ эВ, перестали образовываться электроны и позитроны. Те же, что уже были, почти полностью уничтожились за счет аннигиляции, оставив в «живых» ровно столько электронов, сколько до этого сохранилось протонов, чтобы сбалансировать их положительный электрический заряд и сделать Вселенную (как и в самом исходном состоянии) электрически нейтральной.

Через 100 с после Большого Взрыва ( $T=10^{9} \mathrm{~K}$, и размеры Вселенной достигли сотен св.л.) протоны и нейтроны начали сливаться в легчайшие ядра водорода $\mathrm{H}$, дейтерия $\mathrm{D}$, гелия ${ }^{3} \mathrm{He},{ }^{4} \mathrm{He}$ и лития ${ }^{7} \mathrm{Li}$ (более тяжелые ядра не могли тогда образоваться из-за отсутствия стабильных ядер с массовыми числами 5 и 8). Кроме водорода, в основном появлялись ядра ${ }^{4} \mathrm{He}$, которые с тех пор составляют около $1 / 4$ барионной массы Вселенной. Оставшиеся невостребованными лишние нейтроны распались в течение нескольких последующих часов и исчезли со сцены. Этот процесс называется первичным ядерным синтезом, а относительная распространенность в космосе легчайших ядер, которая с достаточно высокой точностью измеряется сегодня, служит хорошим тестом для проверки модели Большого Взрыва.

И только спустя $3 \cdot 10^{5}$ лет, когда температура упала до $10^{4} \mathrm{~K}$ и диаметр Вселенной достиг размеров $10^{7}$ св.л. $\left(10^{20}\right.$ км), ядра стали окружаться электронными оболочками и возникли первые легкие атомы водорода и гелия (первые химические элементы). Поскольку средняя энергия к тому времени снизилась до нескольких эВ, энергии фотонов уже не хватало для разрушения атомов, и излучение в виде фотонов отделилось от материи, продолжая остывать (именно отсюда отсчитывает свою историю реликтовое излучение). До этого «пробег» фотонов из-за интенсивного взаимодействия с другими частицами, а затем и атомами, был настолько мал, что фотоны были буквально «привязаны» к материи, и Вселенная, если бы на нее кто-то мог взглянуть со стороны, не светилась, т.е. была невидимой. Теперь же Вселенная стала прозрачной, или видимой.

Когда температура снизилась до $3000 \mathrm{~K}$, гравитационное притяжение между атомами начало 
превосходить их взаимное отталкивание за счет теплового движения. Гравитация, действуя на случайные флуктуации плотности в пространственном распределении атомов (в основном водорода и гелия), стала стягивать материю, формируя первоначальные крупномасштабные структуры и группировки скопления прото-галактик, на основе которых позднее (через $10^{8}$ лет после Взрыва при температуре в сотни К) стали образовываться звезды и звездные системы - галактики. Изначальные флуктуации плотности сейчас можно наблюдать в виде очень небольшой анизотропии в наблюдаемом угловом распределении реликтового излучения.

\section{«СТАНДАРТНАЯ» РАСПРОСТРАНЕННОСТЬ НУКЛИДОВ}

Основным и единственным источником тестирования теорий эволюции нуклидов является их распространенность, а именно, зависимость относительного количества того или иного нуклида либо от атомного номера $Z$ (числа протонов), либо от числа нейтронов $N$, либо от массового числа $A=N+Z$ (атомного веса). Источниками информации об относительной распространенности нуклидов является земная кора, метеориты, атмосфера Солнца (совместно с короной и солнечным ветром), атмосферы планет, лунный грунт, атмосферы звезд, межзвездная среда, планетарные туманности, галактики, квазары, космические лучи.

Распространенность стабильных нуклидов была получена нами с использованием всех литературных данных об их содержании в атмосфере Солнца, в земной коре и метеоритах Содержания нуклидов относятся к прародительскому веществу солнечной системы и поэтому при нахождении их первоначальной распространенности был учтен радиоактивный распад, который мог привести к изменению в содержании родительских и дочерних нуклидов. Относительные содержания для большинства стабильных нуклидов бралось из [6]. К нашему удивлению, они полностью совпали с современными данными [7, 10]. Эта распространенность может быть принята в качестве «стандартной» распространенности и она приведена на рис.1 (содержания приведены в относительной шкале $\lg \varepsilon(\mathrm{H})=12,0)$.

Для кривой распространенности химических элементов характерно: экспоненциональное уменьшение относительного содержания нуклидов с ростом массового числа вплоть до $A=100$, а затем наблюдается значительное замедление. Наблюдаются большие флуктуации в содержании легких элементов и пики распространенности нуклидов с определенными массовыми числами (магическими числами). Эти особенности должны быть объяснены теорией образования и эволюции нуклидов.

В табл.1 приведены содержания нуклидов для солнечной системы, определенные по метеоритам и пробам земной коры, отношения содержания которых можно определить в атмосферах, по крайней мере,

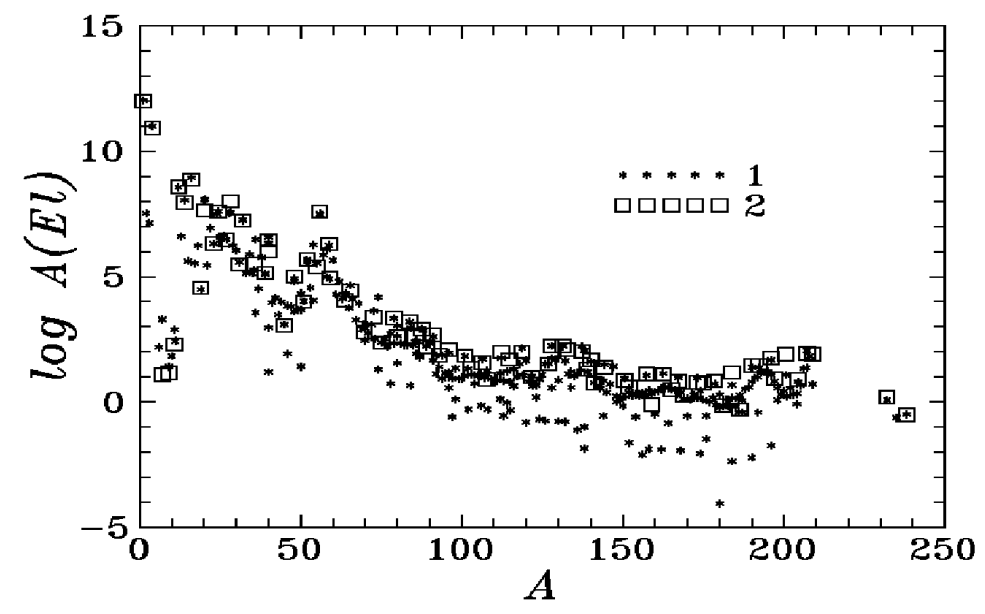

Рис.1. «Стандартная» распространенность нуклидов 
Таблица 1. Содержание нуклидов.

\begin{tabular}{|c|c|c|c|c|c|c|c|}
\hline$Z$ & Нуклид & $A$ & $\begin{array}{c}\text { Распростра- } \\
\text { ненность }\end{array}$ & $Z$ & Нуклид & $A$ & $\begin{array}{c}\text { Распростра- } \\
\text { ненность }\end{array}$ \\
\hline \multirow[t]{2}{*}{1} & $\mathrm{H}$ & 1 & 12.00 & \multirow[t]{4}{*}{20} & \multirow[t]{4}{*}{$\mathrm{Ca}$} & 43 & 3.53 \\
\hline & $\mathrm{D}$ & 2 & 7.22 & & & 44 & 4.69 \\
\hline \multirow[t]{2}{*}{2} & $\mathrm{He}$ & 3 & 7.08 & & & 46 & 1.89 \\
\hline & $\mathrm{He}$ & 4 & 10.99 & & & 48 & 3.64 \\
\hline \multirow[t]{2}{*}{3} & $\mathrm{Li}$ & 6 & 2.22 & \multirow[t]{5}{*}{22} & \multirow[t]{5}{*}{$\mathrm{Ti}$} & 46 & 3.85 \\
\hline & $\mathrm{Li}$ & 7 & 3.31 & & & 47 & 3.82 \\
\hline \multirow[t]{2}{*}{5} & $\mathrm{~B}$ & 10 & 1.83 & & & 48 & 4.82 \\
\hline & B & 11 & 2.43 & & & 49 & 3.70 \\
\hline \multirow[t]{2}{*}{6} & $\mathrm{C}$ & 12 & 8.60 & & & 50 & 3.68 \\
\hline & & 13 & 6,67 & \multirow[t]{4}{*}{26} & \multirow[t]{4}{*}{$\mathrm{Fe}$} & 54 & 6.29 \\
\hline \multirow[t]{2}{*}{7} & $\mathrm{~N}$ & 14 & 8.00 & & & 56 & 7.50 \\
\hline & & 15 & 5.51 & & & 57 & 5.87 \\
\hline \multirow[t]{3}{*}{8} & $\mathrm{O}$ & 16 & 8.84 & & & 58 & 5.05 \\
\hline & & 17 & 5.41 & \multirow[t]{5}{*}{28} & \multirow[t]{5}{*}{$\mathrm{Ni}$} & 58 & 6.09 \\
\hline & & 18 & 6.15 & & & 60 & 5.67 \\
\hline \multirow[t]{6}{*}{12} & $\mathrm{Mg}$ & 24 & 7.50 & & & 61 & 4.33 \\
\hline & & 25 & 6.60 & & & 62 & 4.82 \\
\hline & & 26 & 6.65 & & & 64 & 4.29 \\
\hline & $\mathrm{Si}$ & 28 & 7.55 & \multirow[t]{5}{*}{40} & \multirow[t]{5}{*}{$\mathrm{Zr}$} & 90 & 2.37 \\
\hline & & 29 & 6.24 & & & 91 & 1.71 \\
\hline & & 30 & 6.07 & & & 92 & 1.89 \\
\hline \multirow[t]{2}{*}{20} & $\mathrm{Ca}$ & 40 & 6.36 & & & 94 & 1.90 \\
\hline & & 42 & 4.18 & & & 96 & 1.11 \\
\hline
\end{tabular}

холодных звезд-гигантов (одни отношения содержания изотопов уже определены, а другие должны быть определены в дальнейшем) [5].

\section{ЯДЕРНЫЕ ПРОЦЕССЫ И ЭВОЛЮЦИЯ НУКЛИДОВ}

Можно отметить три этапа в развитии теории происхождения и эволюции нуклидов.

В 30-е годы развитие получила равновесная теория образования элементов, которая объясняла образование всех элементов Вселенной в результате единовременного акта творения. Возникновение этой теории было обусловлено фактом существования корреляции между распространенностью нуклидов и энергией связи ядра. Однако первые сравнения теоретической и наблюдаемой кривой распространенности показали на невозможность образования всех элементов в однородных условиях при одной и той же температуре и давлении. Эта теория в какой-то мере могла объяснить распространенность элементов с атомными номерами до $A=50$ (кроме ${ }^{7} \mathrm{Li},{ }^{9} \mathrm{Be},{ }^{10} \mathrm{~B}$ и ${ }^{11} \mathrm{~B}$ - число перед обозначением элемента равно массовому числу нуклида). Распространенность элементов железного пика $(A=53-63)$ значительно отличается от предсказаний равновесной теории.

В 40-х годах была развита неравновесная теория нейтронного захвата образования всех нуклидов при расширении Вселенной после Большого Взрыва. Образование нуклидов в результате процесса последовательного захвата нейтронов было прекращено из-за быстрого расширения Вселенной и, как следствие этого, падения температуры и плотности (при этих условиях период полураспада нейтрона равняется 15 минутам). Впервые была показана связь процессов образования элементов и их эволюции с космологией - наукой происхождения и развития Вселенной.

В настоящее время считается и проведены соответствующие расчеты, что при космологическом взрыве могли образоваться лишь самые легкие элементы ${ }^{1} \mathrm{H},{ }^{2} \mathrm{H}(\mathrm{D}),{ }^{3} \mathrm{He},{ }^{4} \mathrm{He}$. Более тяжелые элементы образоваться не могли (за исключением, может быть, ${ }^{7} \mathrm{Li},{ }^{9} \mathrm{Be},{ }^{10} \mathrm{~B}$ и ${ }^{11} \mathrm{~B}$ ). При этом необходимо учитывать и образование всех типов элементарных частиц. 
В 50-е годы было выяснено, что основными термодинамическими котлами для производства более тяжелых нуклидов являются недра звезд, где происходит ядерный синтез [8]. Ядерные процессы, обеспечивающие энергетику звезд в разной стадии эволюции и эволюцию нуклидов, суть следующие:

1. Теория горения водорода в результате протон-протонной реакции (рр-цикл) или углеродноазотный $(\mathrm{CN})$-цикл. Если в звезде есть ядра, возникшие в результате горения гелия, то эти процессы могут дать ядра с массовыми числами от 12 до 23. CN-цикл протекает в более горячих (массивных) звездах, a pр-цикл протекает в сравнительно холодных звездах. Горение водорода происходит при температуре равной приблизительно $8 \cdot 10^{6} \mathrm{~K}$ и выше (CNO-цикл эффективен при температурах выше $20 \cdot 10^{6} \mathrm{~K}$ ).

2. Горение гелия ( $3 \alpha$-процесс) в результате приводит к образованию ядер углерода. Для этой реакции необходимы температуры порядка $10^{8}$. Такие условия могут быть только в недрах красных гигантов. При этих условиях путем последовательного захвата $\alpha$-частиц (ядра гелия) могут образоваться ядра до $\mathrm{Mg}$, а затем процесс должен прерваться из-за малой температуры и не возможности преодоления кулоновского барьера

3. Захват $\alpha$-частиц ( $\alpha$-процесс) происходит при температурах в миллиарды градусов. Эти температуры в недрах звезд достигаются после выгорания Не в ядре и его сжатия. При этих температурах энергичные фотоны могут стать источником $\alpha$-частиц, которые в свою очередь синтезируются с ядрами $\mathrm{Ne}$ и образуют ядра $\mathrm{Mg}$. При увеличении температуры до $3 \cdot 10^{9} \mathrm{~K}$ может произойти последовательный захват $\alpha$-частиц и синтезироваться ядра ${ }^{28} \mathrm{Si},{ }^{32} \mathrm{~S},{ }^{36} \mathrm{~A} г$ и ${ }^{40} \mathrm{Ca}$. Это может привести к избытку элементов $\alpha$ процесса. Звезды в это время, как будет сказано ниже, находятся на асимптотической ветви гигантов (АВГ) и их основные параметры не должны зависеть от первоначальной массы, а зависят только от массы вырожденного СО-ядра.

4. При гидростатическом горении $\mathrm{C}, \mathrm{O}$ и $\mathrm{Si}$ выделяется меньше энергии, чем при горении Не (в свою очередь при горении Не выделяется только порядка 10\% энергии по отношению к энергии, выделяемой при горении Н). Звезда эволюционирует в область больших светимостей. Время жизни звезды на этой стадии мало и поэтому маловероятно обнаружить звезду на этой стадии и подвергнуть анализу химический состав ее атмосферы.

5. Равновесный процесс (е-процесс) ответственен за образование элементов в области «железного» типа и он происходит после $\alpha$-процесса, когда звезда снова сжимается и температура достигает $4 \cdot 10^{9} \mathrm{~K}$. Вещество в этом состоянии может пребывать очень короткое время, а затем ядерные процессы должны прерваться (заморозиться). При этих условиях наиболее вероятно образование элементов с наибольшими энергиями связи в ядре, а, именно, ядра с массовыми числами от 50 до 60 (ядра элементов железного пика).

6. Медленный захват нейтронов (s-процесс) ответственен за образование нуклидов с $A=56$ и до $A=209$ ( $\left.{ }^{209} \mathrm{Bi}\right)$. Процесс прерывается из-за образования ядер, подверженных $\alpha$-распаду. Основным параметром s-процесса является источник нейтронов. В недрах красных гигантов предположительно существуют два таких источника: ${ }^{13} \mathrm{C}(\alpha, \mathrm{n}){ }^{16} \mathrm{O}$ или ${ }^{22} \mathrm{Ne}(\alpha, \mathrm{n}){ }^{25} \mathrm{Mg}$. Распространенность нуклидов при sпроцессе должна быть обратно пропорциональна эффективным сечениям захвата нейтронов. Важность процесса захвата нейтрона стала ясна после его открытия.

7. Быстрый захват нейтронов (r-процесс) при котором происходит последовательный захват нескольких нейтронов, прежде чем произойдет радиоактивный распад. Этот процесс возможен лишь при мощном потоке нейтронов. Такие потоки возможны только при вспышках сверхновых звезд. Так как поток нейтронов быстро падает после взрыва, то время быстрой цепной реакции захвата нейтронов не превышает 100 с. При r-процессе образуются богатые нейтронами тяжелые элементы до $A=270$. Ядра с $A=260-270$ не устойчивы и распадаются на осколки и этот процесс происходит быстрее, чем захваты нейтронов и поэтому r-процесс обрывается.

8. Захват протонов (р-процесс) ответственен за образование элементов с избытком протонов $\left({ }^{74} \mathrm{Se}\right.$, ${ }^{78} \mathrm{Kr},{ }^{44} \mathrm{Sr},{ }^{92} \mathrm{Mo},{ }^{94} \mathrm{Mo},{ }^{96} \mathrm{Ru},{ }^{102} \mathrm{Pd},{ }^{106} \mathrm{Cd}$ и т.д.). Богатые протонами ядра могут образоваться в некоторых 
фазах вспышек сверхновых.

9. Бомбардировки ядер C,N,O протонами и $\alpha$-частицами высоких энергией (1-процесс). Легкие ядра ${ }^{6} \mathrm{Li},{ }^{7} \mathrm{Li},{ }^{9} \mathrm{Be},{ }^{10} \mathrm{~B}$ и ${ }^{11} \mathrm{~B}$ не могут образоваться в процессе звездного ядерного синтеза (за исключением, может быть, ${ }^{7} \mathrm{Li}$ при условии его быстрого выноса в области звезды с более низкой температурой), а наоборот разрушаются в звездных недрах ( $\mathrm{Li}$ при температуре в $2 \cdot 10^{6} \mathrm{~K}$, Ве при температуре в $4 \cdot 10^{6} \mathrm{~K}$, а $\mathrm{B}$ при температурах более $5 \cdot 10^{6} \mathrm{~K}$ ). Поэтому их содержание в атмосферах звезд должно быть в 100 раз ниже первоначальных значений (после Большого Взрыва?). Однако у некоторых звезд-гигантов содержание ${ }^{7} \mathrm{Li}$ превосходит космологическое содержание. Содержания ${ }^{7} \mathrm{Li}$ в метеоритах и в атмосфере Солнца различаются почти на два порядка. В настоящее время считается, что легкие элементы образовались в результате бомбардировки галактическими космическими лучами межзвездной среды. В результате 1процесса могли образоваться ядра ${ }^{6} \mathrm{Li},{ }^{9} \mathrm{Be},{ }^{10} \mathrm{~B}$ и ${ }^{11} \mathrm{~B}$. Перед астрофизиками стоит проблема определение содержания легких элементов в атмосферах наиболее старых звезд с дефицитом металлов. Они должны иметь космологическое содержание этих элементов.

Все эти процессы качественно могут объяснить распространенность нуклидов во Вселенной, в частности, в солнечной системе и в атмосферах звезд.

\section{НУКЛИДЫ В ЗВЕЗДАХ}

Первые звезды, по всей вероятности, состояли практически только из водорода и гелия в виде горячей плазмы с температурой в центральной части, достаточной для протекания термоядерных реакций, в результате которых образовались более тяжелые элементы - вплоть до железа. Химические элементы тяжелее железа рождались в результате взрыва сверхновых звезд. Чем больше масса звезды, тем меньше она живет. По мере «выгорания» термоядерного топлива в достаточно массивной звезде $\left(M>10 M_{\odot}\right)$ силы гравитационного притяжения приводят к схлопыванию звезды - гравитационному коллапсу, когда звезда с огромной скоростью начинает сжиматься к центру. Выделяемая энергия не успевает покинуть звезду, и она взрывается. В результате такого взрыва образуются новые, более компактные объекты в виде белых карликов, нейтронных звезд или черных дыр и выделяется колоссальная энергия, большую часть которой уносят нейтрино. В космическое пространство с огромной скоростью разлетается газообразное облако остатков прежней звезды, привнося в космос новые химические элементы. Именно поэтому более поздние звездные образования, включая наше Солнце и планеты Солнечной системы, имеют полный набор элементов периодической таблицы Менделеева. Кроме того, в звездах образуется вся совокупность нуклидов как стабильных, так и нестабильных. Нестабильные нуклиды играют основную роль в температурном режиме и, следовательно, в эволюции протозвездного и протопланетного вещества (включая солнечную систему). Основной задачей современной астрофизики в изучении звезд является определение содержания химических элементов и изотопов в их атмосферах, в частности, холодных звезд-гигантов и сверхгигантов, с целью тестирования теорий эволюции нуклидов. Они обладают высокими светимостями и доступны исследованию даже при значительном удалении от наблюдателя. К тому же спектры этих звезд имеют множество атомных и молекулярных линий поглощения, которые образуются на разных глубинах их атмосфер [5]. Это дает принципиальную возможность исследовать структуру и физико-химические параметры атмосфер $\mathrm{G}, \mathrm{K}, \mathrm{M}$ - гигантов в зависимости от их расположения не только в нашей Галактике, но и в ближайших галактиках, позволяет надежно определить содержание химических элементов и их изотопов в атмосферах. В свою очередь это позволяет тестировать теории эволюции звезд и эволюции стабильных и даже некоторых радиоактивных нуклидов (по крайней мере, с периодами полураспада $\sim 10^{5}$ лет). В последнее время, особое значение приобретает определения содержания химических элементов в атмосферах старых звезд с малой массой (субкарликов карликов и «коричневых» карликов), атмосферы которых должны содержат информацию о составе прародительского вещества звезды. В процессе эволюции галактического тонкого и толстого дисков следующее поколение звезд связано с предыдущим через межзвездную среду, которая в процессе 
эволюция обогащается продуктами звездного термоядерного синтеза и который происходит в недрах прародительских звезд. По современным представлениям теории эволюции звезд время пребывания звезды на определенной стадии (в том или ином месте диаграммы Герцшпрунга-Рессела) существенно зависит от ее массы, первоначального химического состава и процессов термоядерного синтеза. Поэтому определение химического состава атмосфер звезд дает информацию о химической эволюции диска и гало Галактики, так как возраст звезд простирается от самой ранней эпохи возникновения Галактики до настоящего времени. Они и содержат запись состава нуклидов в разные эпохи эволюции Галактики. Изучение эволюции нуклидов проливает свет на эволюции всех объектов Вселенной. Определение химического состава атмосфер звезд и межзвездной среды играет определяющую роль при анализе всех процессов, включая процессы термоядерного синтеза и радиоактивного распада, происходящих при эволюции Галактики. Звезды диска, гало, балджа (центрального утолщения) и бара (перемычки) Галактики находятся на разных стадиях эволюции - первая и последующие ветви гигантов (ВГ), голубая и красная части горизонтальной ветви гигантов (ГВГ и КВГ), асимптотическая ветвь гигантов (АВГ), и они расположены в проникающих в друг друга областях диаграммы ГР. Если теория эволюции звезд в плане перемешивания вещества их атмосфер с продуктами термоядерного синтеза имеет под собой основание, то содержания химических элементов и их изотопов в атмосферах холодных звезд-гигантов и сверхгигантов при надежном определения их фундаментальных характеристик может дать информацию об эволюционном статусе звезды, т.е. об ее массе и процессах «загрязнения» прародительского вещества их атмосфер продуктами термоядерного синтеза.

Предполагается существование трех этапов возможного глубокого перемешивания:

- на стадии ГП, или при выходе на стадию ВГ;

- при выходе на стадию АВГ (во время горении Н и Не в слоевых источниках);

- во время гелиевых вспышек в конце АВГ.

Итак, расчеты показывают, что звезда, попадая на ветвь гигантов, может испытать первое глубокое перемешивание из-за быстрого распространения конвективной зоны вглубь звезды. Более того, перемешивание может произойти даже на стадии ГП, хотя механизмы такого перемешивания не совсем ясны (меридиональная циркуляция, турбулентная диффузия, вращение).

Среди звездного населения Галактики наблюдаются так называемые углеродные звезды, в спектрах которых интенсивны молекулярные полосы соединений углерода. Углеродные звезды эволюционируют вдоль АВГ. При достижении верхней части первой ветви гигантов возможна вторая вспышка перед выходом звезды на вторую ветвь гигантов или на асимптотическую ветвь, так называемая гелиевая вспышка. Она обусловлена развитием тепловой неустойчивости в вырожденном ядре звезды. В результате вспышки гелиевое ядро становится невырожденным и снова наступает спокойная стадия горения гелия в ядре. Такая эволюция характерна для звезд малой массы $\left(M<2,25 M_{\odot}\right)$. У звезд промежуточных масс $\left(M<8 M_{\odot}\right)$ вырождение в центральных областях наступает только после образования углероднокислородного ядра и двух слоевых источников энергии звезды: гелиевого и водородного. Эволюция звезд в этой стадии удивительно похожа как для звезд малых масс, так и для звезд промежуточных масс. На ранней стадии АВГ водородный слой практически неактивен и большая часть энергии выделяется в гелиевом слое. Он находится непосредственно над углеродно-кислородным ядром. Масса водородного слоя практически не меняется. Гелиевый источник по мере выгорания движется наружу. На этой стадии не происходит изменения химического состава атмосфер звезд. Когда активный слой горения гелия приблизится к водородному слою, в нем начинают происходить ядерные реакции. При этом гелиевый слой начинает пульсировать. Это приводит к начальному этапу нахождения звезды на АВГ и тогда в гелиевом слое начинаются тепловые вспышки. Причина вспышек - это накопление энергии у нижнего дна водородной зоны в результате расширения гелиевой зоны. Скорость выделения энергии в результате $\alpha$ процесса превосходит скорость выноса энергии наружу излучением. Происходит стремительное выделение энергии - тепловая вспышка в гелиевом слоевом источнике. Избыток энергии вызывает конвекцию в 76 Комаров H.C. 
тонком слое над областью горения гелия. Это вызывает его расширение до водородного слоевого источника и охлаждение. Водородный источник снова становится неактивным. Происходит охлаждение и гелиевого слоевого источника и вещество звезды снова сжимается. Это приводит к возрождению ядерных реакций в гелиевом слоевом источнике. Отметим, что положение звезды на диаграмме ГР мало зависит от полной массы звезды, а зависит лишь от массы углеродно-кислородного ядра. Звезды в среднем движутся по одному и тому же треку (поэтому и называется асимптотической ветвью гигантов). Как сказано выше, движение по этому треку сопровождается многократными тепловыми вспышками в гелиевом слоевом источнике энергии.

Существенным вопросом при эволюции звезд на стадии АВГ - это вопрос о темпе потери их массы. Дальнейшая эволюция этих звезд зависит от скорости потери массы, а именно, станут ли они белыми карликами или сверхновыми. От этого зависит темп обогащения межзвездной среды теми или иными нуклидами.

Принадлежность звезд к разным типам населения Галактики, к разным типам скоплений и к динамическим группам дает прекрасную возможность проследить эволюцию химического состава их атмосфер в зависимости от возраста и химического состава прародительского вещества. Эволюция звезд разных масс и разного первоначального химического состава происходит по разному сценарию.

\section{А) Нуклиды CNO-процесса.}

Холодные звезды главной последовательности (ГП) диска Галактики в окрестности Солнца имеют средние содержания элементов СNO-группы близкие к солнечному, а именно, $[\mathrm{C} / \mathrm{H}]=-0,03+0,1$, $[\mathrm{N} / \mathrm{H}]=0,02+0,1$ и $[\mathrm{O} / \mathrm{H}]=0,00+0,1$, где $[\mathrm{El} / \mathrm{H}]=\lg (\mathrm{El} / \mathrm{H})^{*}-\lg (\mathrm{El} / \mathrm{H})^{\mathrm{o}}, \lg (\mathrm{El})^{*}-$ содержание определенного химического элемента в атмосфере звезды, а $\lg (\mathrm{El})^{\circ}$ содержание того же элемента в атмосфере Солнца. Логарифм содержания водорода принимается равным 12,0. В тоже время содержания металлов может меняться в пределах от 0,3 до -0,7 dex. Средний уровень металличности может меняться от одного рассеянного скопления к другому. Четкой зависимости от возраста не обнаружено. Поэтому необходимо сравнивать содержания, полученные разными авторами, по отношению к железу, как наиболее стабильному элементу (наибольшая энергия связи ядра). Получено, что в атмосферах звезд-карликов в спектральном диапазоне от $\mathrm{F}$ до ранних $\mathrm{K}$ средние значения $[\mathrm{El} / \mathrm{Fe}]$, в частности от $\mathrm{C}$ до $\mathrm{Sc}$, находятся в пределах от 0,08 до -0,04 dex. Прародителями звезд-гигантов спектральных классов $\mathrm{G}-\mathrm{M}$ являются звезды-карлики спектральных классов F-K и они имеют массы в пределах от 3 до 0,8 $M_{\odot}$. Поэтому нахождение содержания нуклидов в их атмосферах может дать информацию как о процессах термоядерного синтеза в недрах звезд, так и о возможных механизмах переноса вещества из недр на поверхность. Разбавлено ли прародительское вещество в их атмосферах продуктами термоядерного синтеза? Действительно, для 4-х и 2-х звезд-гигантов скоплений Гиад и Ясли среднее отношение $\mathrm{C} / \mathrm{N}=0,9$, а для Солнца $\mathrm{C} / \mathrm{N}=4,8$. При этом для звезд-карликов скопления Гиад отношение содержания углерода к азоту такое же, как и для Солнца. В среднем для звезд-гигантов поля диска Галактики получено, что $\mathrm{C} / \mathrm{N}=2,3$, т.е. содержание азота растет, а углерода уменьшается. Эта аномалия увеличивается для звезд-гигантов с большей светимостью (с изменением $\mathrm{M}_{\mathrm{V}}$ от 3,5 до 2,8 отношение $\mathrm{C} / \mathrm{N}$ падает в четыре раза). При дальнейшем увеличении светимости величина $\mathrm{C} / \mathrm{N}$ остается приблизительно одинаковой и равной $\mathrm{C} / \mathrm{N}=1$. Для звезд с дефицитом металлов и с массами меньшими массы Солнца отношение содержания углерода к азоту равно солнечному, в то время как для звезд-гигантов отношения содержания азота к железу выше, а отношение содержания углерода к железу меньше, чем в атмосфере Солнца. В тоже время суммарное содержание элементов $\mathrm{C}, \mathrm{N}, \mathrm{O}$ для звезд-карликов и звезд- гигантов скопления Гиад и звездного поля диска Галактики почти одинаково. Таким образом, для атмосфер звезд-гигантов характерно уменьшение содержания углерода, увеличение содержания азота при почти постоянном содержании кислорода по сравнению с содержанием их в атмосферах звезд-карликов. Для звезд-гигантов с экстремально низким содержанием металлов обнаружена тенденция уменьшения содержания С и увели- 
чение содержания $\mathrm{N}$ по отношению к $\mathrm{Fe}$ до 10 раз. Отметим, что теория эволюции одиночных звезд предсказывает после перемешивания более высокое отношение $\mathrm{C} / \mathrm{N}=2$. Это указывает на неизвестные механизмы перемешивания. Итак, когда звезда попадает на ветвь гигантов, изменяется лишь относительное количество нуклидов C, N, O, а также содержание натрия [2,5].

Дополнительным доказательством выноса продуктов горения водорода на поверхность звезды является отношение содержания изотопов углерода ${ }^{12} \mathrm{C} /{ }^{13} \mathrm{C}$. Интересно отметить, что вынос продуктов термоядерного синтеза на поверхность звезды зависит от ее массы. Для звезд с $M>2 M_{\odot}$ значения ${ }^{12} \mathrm{C} /{ }^{13} \mathrm{C}$ находятся в пределах от 20 до 30. Эти значения хорошо согласуются с теорией первого глубокого перемешивания. Другая картина наблюдается для звезд с $M<2 M_{\odot}$. Отношение содержания нуклидов ${ }^{12} \mathrm{C} /{ }^{13} \mathrm{C}$ значительно уменьшается при уменьшении массы звезды и это противоречит теоретическим расчетам. Для согласования теории и наблюдений приходится предположить существование неизвестного механизма перемешивания, который может быть эффективен даже на стадии ГП. После выгорания гелия в ядре звезды (конечная стадия первой ветви гигантов) химический состав ее атмосферы не должен отличаться от начального. На стадии АВГ химический состав атмосфер звезд будет зависеть от температуры у основания конвективной оболочки. Если температура будет ниже некоторого значения, то углерод не подвергается реакциям CNO-цикла, при которых ${ }^{12} \mathrm{C}$ превращается в ${ }^{14} \mathrm{~N}$ с образованием ${ }^{13} \mathrm{C}$. Для большинства звезд при эволюции вдоль АВГ вынос продуктов ядерного синтеза увеличивает содержание нуклида ${ }^{12} \mathrm{C}$.

Для тестирования ядерных процессов особую роль играют изотопы кислорода

Содержания нуклида ${ }^{16} \mathrm{O}$ почти не меняется из-за малости температуры для реакции ${ }^{13} \mathrm{C}(\alpha, \gamma){ }^{16} \mathrm{O}$. Это означает, что отношения нуклидов ${ }^{12} \mathrm{C} /{ }^{16} \mathrm{O}$ и ${ }^{12} \mathrm{C} /{ }^{13} \mathrm{C}$ должны расти, а содержания нуклидов ${ }^{14} \mathrm{~N}$ и ${ }^{16} \mathrm{O}$ не должны изменяться. В атмосферах звезд отношения содержания этих нуклидов должны изменяться еще при переходе звезды на ветвь гигантов во время первого и второго перемешивания. Для атмосферы Солнца ${ }^{16} \mathrm{O} /{ }^{17} \mathrm{O}=2700{ }^{16} \mathrm{O} /{ }^{18} \mathrm{O}=490$ [11]. В процессе эволюции звезды после первого глубокого перемешивания происходит уменьшение отношения содержания нуклидов ${ }^{16} \mathrm{O} /{ }^{17} \mathrm{O}$ до значений от 300 до 1000 , что качественно подтверждается и наблюдениями спектров холодных звезд-гигантов [12], в тоже время как отношение ${ }^{16} \mathrm{O} /{ }^{18} \mathrm{O}$, практически совпадает с солнечным содержанием. Во время второго перемешивания содержания нуклидов ${ }^{16} \mathrm{O}$ и ${ }^{18} \mathrm{O}$ не должно сильно изменяться. Изменение содержания нуклида кислорода ${ }^{17} \mathrm{O}$ зависит от скорости реакции ${ }^{17} \mathrm{O}(\mathrm{p}, \alpha){ }^{14} \mathrm{~N}$.

Большое внимание было уделено определению отношения содержания нуклидов ${ }^{12} \mathrm{C} /{ }^{13} \mathrm{C}$. Звезды на АВГ имеют эти отношения в интервале от 20 до 100. Это отражает увеличение нуклида ${ }^{12} \mathrm{C}$ при почти постоянном значении содержания нуклида ${ }^{13} \mathrm{C}$. В гигантах до стадии АВГ это отношения находятся в пределах от 10 до 20. Наличие молекулярных линий и полос углеродосодержащих молекул дает возможность определить изотопный состав углерода, в частности, отношения в содержании ${ }^{12} \mathrm{C} /{ }^{13} \mathrm{C}$. Как было показано выше, в атмосферах холодных звезд-гигантов отношение ${ }^{12} \mathrm{C} /{ }^{13} \mathrm{C}$ может меняться в пределах от 4 до 40, что уже само по себе говорит об изменениях в химическом составе атмосфер звезд в результате выноса на поверхность продуктов термоядерного синтеза. Эти изменения обусловлены выносом продуктов горения водорода в атмосферу звезды в результате первого перемешивания, которое может при некоторых условиях произойти и на стадии ГП. Эти условия прежде всего определяются первоначальной массой звезды и прародительским химическим составом межзвездной среды. Однако существуют сверхгиганты с отношением ${ }^{12} \mathrm{C} /{ }^{13} \mathrm{C}=4$, что также требует дополнительных механизмов перемешивания.

В тоже время, для поддержания структуры красного гиганта необходимо отсутствие полного перемешивания между внутренними и внешними слоями, что приводит к необходимости разработки особых механизмов выноса продуктов термоядерного синтеза в их атмосферы, в частности, на стадии АВГ предполагается попеременное проникновение слоев горения Н и Не в приграничные слои, которое возможно и обеспечивает вынос продуктов горения на поверхность, что и приводит к повышению содержания ${ }^{12} \mathrm{C}$.

Отношения содержания нуклидов элементов $\mathrm{C}, \mathrm{O}, \mathrm{N}, \mathrm{Mg}, \mathrm{Al}, \mathrm{Si}, \mathrm{Ca}, \mathrm{Ni}, \mathrm{Zr}$ могут отличаться от 
«стандартных» и дать информацию о термоядерных процессах присоединения $\alpha$-частиц и нейтронов. Как было показано выше, отношения содержания нуклидов ${ }^{12} \mathrm{C} /{ }^{13} \mathrm{C}$ для звезд-гигантов значительно меньше земного (солнечного). Особый интерес представляют красные гиганты с дефицитом металлов (с металличностью равной $-2,0$ dex), которые должны быть звездами с малой массой и образоваться из облака с массой равной $10^{6}$ и $10^{7} M_{\odot}$. Массивные короткоживущие звезды поставляют в это облако различные металлы и продукты CNO-цикла. Вариации интенсивностей полос CN, CH и NH свидетельствует о том, что красные гиганты произошли из прародительского вещества с разным соотношением продуктов термоядерного синтеза.

В нормальных гигантах в процессе термоядерного синтеза можно ожидать изменения отношений в содержании нуклидов в результате двойственности (бариевые звезды), вынужденной циркуляции, гелиевых вспышек, глубоко проникающей конвекции и потери массы эти изменения могут проявиться на поверхности звезды в разных соотношениях.

При дальнейшей эволюции звезд с $M>1.5 M_{\odot}$ возможно звезда последовательно проходит спектральные классы M-MS-S-SC-C (R и N). Эта последовательность обусловлена прежде всего изменением

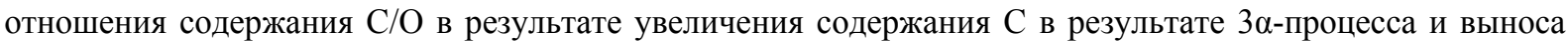
продуктов этого процесса в атмосферу звезд. Для S и SC (циркониевых и циркониево-углеродных) звезд отношение $\mathrm{C} / \mathrm{O}=1$, а для $\mathrm{C}(\mathrm{N}, \mathrm{R})$ (углеродных) - $\mathrm{C} / \mathrm{O}>1$. Половина $\mathrm{N}$-звезд имеют значения $\mathrm{C} / \mathrm{O}$ в интервале от 1,01 до 1,10 .

Звезды спектрального класса $\mathrm{N}$ (в настоящее время звезды C не разделяются на $\mathrm{N}$ и $\mathrm{R}$, хотя они имеют разный эволюционный статус) принадлежат АВГ и среди этих звезд имеются звезды с отношением нуклидов ${ }^{12} \mathrm{C} /{ }^{13} \mathrm{C}=80 \div 90$ и звезды с отношением изотопов ${ }^{12} \mathrm{C} /{ }^{13} \mathrm{C}=3 \div 4$ (такие звезды выделены в особый класс J-звезд и в их атмосферах не обнаружен избыток элементов s-процесса). Звезды спектрального класса $\mathrm{R}$ не относятся к АВГ и поэтому их фундаментальные параметры и химический состав атмосфер отличен от $\mathrm{N}$-звезд. Так для N-звезд интервалы эффективных температур и логарифмов ускорений силы тяжести заключены в пределах от $2300 \mathrm{~K}$ до $3200 \mathrm{~K}$ и от $-1,1$ до 0,3, а для R-звезд - от $4200 \mathrm{~K}$ до $5000 \mathrm{~K}$ и от 2,0 до 2,2. Итак, звезды на АВГ, которые возможно подвержены неоднократным вспышкам в гелиевом слоевом источнике, имеют атмосферы с избытком изотопа ${ }^{12} \mathrm{C}$ по отношению к холодным звездам-гигантам спектральных классов G, K, но приблизительно равное по отношению к Солнцу.

\section{Б) Нуклиды l-процесса.}

Содержание легких элементов - это ключ к пониманию таких существенных проблем, как: эволюция Вселенной, галактик, происхождения космических лучей, эволюции звезд и межзвездной среды, эволюции протосолнечной ОВ-ассоциации (если такова существовала), химической эволюции всех частей Галактики.

Легкие элементы Li, Be, В легко разрушаются при низких температурах. Так как при переходе от ГП к звездам-гигантам происходит увеличение температуры, то следует ожидать уменьшения в содержании этих легких элементов по отношению к их «космологическому» содержанию. Действительно содержание ${ }^{7} \mathrm{Li}$ в атмосфере Солнца мало по сравнению с его содержанием в метеоритах и в образцах земной породы, но в тоже время содержание ${ }^{7} \mathrm{Li}$ в метеоритах превосходит «космологическое» содержание. И в тоже время имеются красные гиганты с избытком ${ }^{7} \mathrm{Li}$. Содержание Be в атмосфере Солнца близко к метеоритному содержанию и содержанию в земной коре. Вновь образовавшиеся звезды имеют содержание ${ }^{7} \mathrm{Li}$ близкое к метеоритному. Так в атмосферах звезд типа $\mathrm{T}$ Tau $\lg \mathrm{A}(\mathrm{Li})=3 \div 4$. В скоплении Плеяды (возраст $3 \cdot 10^{7}$ лет) звезды-карлики спектрального класса F (гигантов еще нет) имеют содержание лития приблизительно равное 3,3 dex, а для звезд-карликов спектрального класса $\mathrm{K}-2,6$ dex. Для поздних звезд, принадлежацих скоплению Гиад (возраст $4 \cdot 10^{8}$ лет), содержание лития для звезд равно $\lg (\mathrm{Li})=1,5$. Содержания Li в атмосферах разных звезд может различаться на шесть порядков. Содержания Ве и В в атмосферах большинства звезд вполне согласуется с содержанием их в атмосфере Солнца.

Легкие ядра $\mathrm{Li}, \mathrm{Be}$, и В не могут образоваться в процессе звездного термоядерного синтеза за ис- 
ключением, может быть, ${ }^{7} \mathrm{Li}$ при условии быстрого выноса изотопа ${ }^{7} \mathrm{Be}$ в области звезды с более низкой температурой в результате ядерной реакции ${ }^{3} \mathrm{He}(\alpha, \gamma)^{7} \mathrm{Be}(\mathrm{e}, v)^{7} \mathrm{Li}$. Эти условия могут осуществиться при эволюционной стадии красного гиганта во время вспышек. При этом происходит частичное перемешивание зоны горения $\mathrm{H}$, где может быть значительная концентрация ${ }^{3} \mathrm{He}$, с зоной богатой ${ }^{4} \mathrm{He}$. Все остальные легкие нуклиды разрушаются в звездных недрах (Li при температуре в $2 \cdot 10^{6} \mathrm{~K}$, Be при температуре в $4 \cdot 10^{6} \mathrm{~K}$, а В при температурах более $5 \cdot 10^{6} \mathrm{~K}$ ). Поэтому их содержание в атмосферах звезд должно быть в 100 раз ниже первоначальных значений (после Большого Взрыва). Однако, как показано выше, у некоторых звезд-гигантов содержание ${ }^{7} \mathrm{Li}$ превосходит космологическое содержание. Содержания ${ }^{7} \mathrm{Li}$ в метеоритах и в атмосфере Солнца различаются почти на два порядка. В настоящее время считается, что легкие нуклиды в основном образовались в результате бомбардировки галактическими космическими лучами межзвездной среды. В результате 1-процесса могли образоваться ядра ${ }^{6} \mathrm{Li},{ }^{7} \mathrm{Li},{ }^{9} \mathrm{Be},{ }^{10} \mathrm{~B}$ и ${ }^{11} \mathrm{~B}$. Отметим, что распространенность элементов $\mathrm{Li}, \mathrm{Be}$ и В в галактических космических лучах на $4 \div 5$ порядков выше, чем в атмосферах звезд. Перед астрофизиками стоит проблема определение содержания легких элементов в атмосферах наиболее старых звезд с дефицитом металлов (хотя в настоящее время дефицит металлов не является признаком старости звезд). Необходимо оценить космологическое содержание этих элементов, наблюдая самые старые звезды Галактики. По всей вероятности, мы должны учитывать возможность образования ${ }^{7} \mathrm{Li}$ в недрах холодных звезд-гигантов и при взрывах новых. Для объяснения выноса ${ }^{7} \mathrm{Li}$ на поверхность привело к разработке альтернативных теорий перемешивания (восходящие струи, диффузия и т.д.). Эти теории в какой-то мере могут объяснить вынос на поверхность звезд-гигантов нуклидов ${ }^{13} \mathrm{C},{ }^{15} \mathrm{~N},{ }^{17} \mathrm{O}$ и s-процесса. Отметим, что содержание Li коррелирует с отношением в содержании нуклидов ${ }^{12} \mathrm{C} /{ }^{13} \mathrm{C}$, а именно, звезды-гиганты с высоким содержанием изотопа ${ }^{13} \mathrm{C}$ имеют низкое содержание $\mathrm{Li}$. Таким образом, содержание изотопа ${ }^{13} \mathrm{C}$ и ${ }^{7} \mathrm{Li}$ позволяет грубо оценить массу звезды и, следовательно, и ее возраст. Однако имеются звезды в атмосферах которых определены и высокое содержание $\mathrm{Li}$, и низкие отношения $\mathrm{C} / \mathrm{N}$ и ${ }^{12} \mathrm{C} /{ }^{13} \mathrm{C}$, что подтверждает вывод о возможности синтеза Li в недрах звезд с последующим выносом на поверхность. Эти звезды имеют массы $M<1,5 M_{\odot}$, для которых возможна вспышка Не-ядра.

В этом отношении показателен объект Кассиопея A - остаток сверхновой, вспыхнувшей примерно 300 лет назад. Обнаружены волокна с преимущественным содержанием кислорода. Видны линии элементов серы, аргона и кальция. Это означает, что звезда на эволюционной стадии предсверхновой имела слоистое строение и толщины этих слоев зависят от первоначальной массы звезды.

\section{В) Нуклиды $\alpha$ - и s-процессов}

Были проведены оценки отношений в содержании изотопов Ті по полосам поглощения (линиям поглощения) молекулы окиси титана ТіО. Расчеты синтетических спектров с различным изотопным составом показали, что редкие изотопы $\mathrm{Ti}\left({ }^{46} \mathrm{Ti},{ }^{47} \mathrm{Ti},{ }^{49} \mathrm{Ti},{ }^{50} \mathrm{Ti}\right)$ присутствуют в атмосферах холодных звезд разной светимости и эти отношения для большинства звезд близки к солнечным. Другую группу измеренных отношений изотопов составляют элементы $\mathrm{Mg}$ (по полосам молекулы $\mathrm{MgH}$ ), $\mathrm{Zr}$ (по полосам молекулы $\mathrm{ZrO}$ ). Они также близки к солнечным ${ }^{24} \mathrm{Mg} /{ }^{25} \mathrm{Mg} /{ }^{26} \mathrm{Mg}=79 / 10 / 11$ [12]. Томкин и Ламберт [16] исследовали содержание изотопов магния в атмосферах бариевой и умеренно бариевой звезд и обнаружили солнечное отношение изотопов $\mathrm{Mg}$. В тоже время в атмосфере старой звезды-субкарлика класса $\mathrm{K}$ Грумбридж $1830{ }^{24} \mathrm{Mg} /{ }^{25} \mathrm{Mg} /{ }^{26} \mathrm{Mg}=94 / 7 / 3$. Лишь при этом соотношении изотопов наблюденный спектр согласуется с синтетическим. Это согласие соблюдается даже при $100 \%$ содержании ${ }^{24} \mathrm{Mg}$. В атмосфере этой же звезды наблюдается дефицит в содержании элементов с избытком нейтронов $\left({ }^{23} \mathrm{Na},{ }^{25} \mathrm{Mg},{ }^{26} \mathrm{Mg}\right.$, $\left.{ }^{27} \mathrm{Al}\right)$ по отношению к элементам $\alpha$-процесса $\left({ }^{24} \mathrm{Mg}\right)$, а так же дефицит в содержании элементов группы $\mathrm{Fe}$ $([\mathrm{Fe} / \mathrm{H}]=-1.37)$. Эти результаты позволяют предположить, что источником нейтронов для s-процесса является реакция ${ }^{13} \mathrm{C}(\alpha, \mathrm{n}){ }^{16} \mathrm{O}$.

Весьма важным для тестирования теории эволюции звезд и для понимания процессов, происходящих в недрах звезд при их эволюции, является сравнение теоретических и наблюденных содержания в 
атмосферах некоторых красных гигантов неустойчивых изотопов ${ }^{26} \mathrm{Al}\left(7,4 \cdot 10^{4}\right),{ }^{93} \mathrm{Zr}\left(1,5 \cdot 10^{6}\right),{ }^{99} \mathrm{Tc}\left(2 \cdot 10^{5}\right)$. В скобках указаны периоды полураспада. Обнаружение линий поглощения этих нуклидов в спектрах звезд на стадии АВГ говорит о недавнем синтезе тяжелых элементов в недрах звезд в результате sпроцесса. Технеций в атмосферах циркониевых звезд открыл Меррил [13].Содержание ${ }^{99}$ Тс в атмосферах звезд, в спектрах которых обнаружены линии поглощения этого элемента, находится в пределах от -1.0 до +1.5 dex. Имеются звезды на стадии АВГ, в спектрах которых линии ${ }^{99}$ Тс не наблюдаются. По данным Смита и Ламберта [15] у 40\% MS и S звезд не обнаружено ${ }^{99} \mathrm{Tc}$. Звезды, не имеющие ${ }^{99} \mathrm{Tc}$, находятся на ранней стадии АВГ. Их предшественниками являются бариевые звезды. В тоже время в атмосферах этих звезд обнаружен избыток в содержании стабильных нуклидов s-процесса. Изотоп ${ }^{93} \mathrm{Zr}$ также обнаружен в атмосферах S-звезд (R Cyg и V Cnc). Радиоактивный распад нуклида ${ }^{93} \mathrm{Zr}$ приводит к образованию стабильного нуклида ${ }^{93} \mathrm{Nb}$. Это отношение в атмосфере звезд зависит от времени, прошедшего после последней вспышки, от скорости перемешивания вещества в звезде и от интервала времени между вспышками. Эти условия меняются от звезды к звезде. В работе [14] показано, что технеций в звездах на АВГ может образоваться путем фоторасщепления ядер и таким образом наличие технеция не обязательно является тестом, проходящего в недрах звезд s-процесса.

Впервые данные о наличии нуклида ${ }^{26} \mathrm{Al}$ были получены при исследовании метеорита Альенде [9], в котором обнаружен избыток нуклида ${ }^{26} \mathrm{Mg}$. Исследования показали, что повышенное содержание ${ }^{26} \mathrm{Al}$ характерно для всей межзвездной среды, а не появилось в протосолнечной туманности в результате взрыва сверхновой. Наиболее вероятным источником ${ }^{26} \mathrm{Al}$ в межзвездной среде являются звезды на стадии АВГ. Во время горения гелия в слоевом источнике происходит выгорание азота, который присутствует в большом количестве. Он образовался в результате ранее проходившего СNO-цикла. В результате этой цепочке реакций все нуклиды углерода и кислорода превратились в азот. В конвективном слое между водородным и гелиевым слоевыми источниками образуется нуклид ${ }^{25} \mathrm{Mg}$. После очередной гелиевой вспышки этот нуклид выносится в водородную оболочку с достаточно высокой температурой для протекания ядерной реакции ${ }^{25} \mathrm{Mg}(\mathrm{p}, \gamma)^{26} \mathrm{Al}$. Хорошее согласие с наблюдениями удалось получить в предположении, что на стадии АВГ потеря массы увеличивается при достижении определенной светимости $(\log \mathrm{L} \sim 4)$. Это говорит о том, что наряду со сбросом планетарной туманности в звездах АВГ происходит дополнительная потеря массы. Таким образом исследовать отношение нуклидов ${ }^{26} \mathrm{Al}$ и ${ }^{27} \mathrm{Al}$ необходимо в атмосферах звезд большой светимости на АВГ. При $M_{\mathrm{bol}} \sim-6$ отношение содержания нуклидов ${ }^{26} \mathrm{Al} /{ }^{27} \mathrm{Al} \sim 1$.

Рассмотрим содержание других элементов s-процесса в атмосферах холодных звезд. Выше было указано, что нуклиды s-процесса могут синтезироваться только в недрах звезд-гигантов и сверхгигантов с $M>1.5 M_{\odot}$. Таким образом, определение содержания химических элементов в атмосферах звезд разных масс дает возможность тестировать правильность эволюционных расчетов. В частности, в работах [2, 35] показано, что атмосферы звезд-гигантов с солнечной металличностью в спектральном диапазоне G5K0 (тонкий диск Галактики) имеют солнечное содержание элементов s-процесса. Звезды спектрального класса K5 имеют избыток тяжелых нуклидов s-процесса. В дальнейшем, было показано, что в атмосфере Альдебарана (динамическая группа Гиады, $\lg t=8.83$ ) содержание Fe и элементов s-процесса солнечное, а в атмосфере Арктура (динамическая группа $\alpha$ Boo, $\lg t=9.6$ ) эти элементы имеют одинаковый дефицит по отношению к их содержанию в атмосферах Солнца и Альдебарана. Однако вопрос о содержании нуклидов s-процесса в звездах тонкого и толстого дисков в интервале спектральных классов К0 и К5 остается открытым (особенно, если учесть распределение средней металличности от спектрального класса и возможной вспышке звездообразования [5]). При новом исследовании использовались линии поглощения в синей области спектра. Из-за большого блендирования и слабости линий поглощения элементов s-процесса определение их содержания сопряжено со значительными случайными и систематическими ошибками (необходимостью проведения уровня «непрерывного» спектра при использовании в методе моделей атмосфер эквивалентной ширины). По крайней мере, требуются 
дополнительные исследования.

В гигантах с большим дефицитом металлов (звездное население гало Галактики) $[\mathrm{Fe} / \mathrm{H}]<-1,3$ содержание нуклидов s-процесса и r,s-процессов должно быть в дефиците по отношению к солнечному содержанию, так как ядра Fе являются зародышевыми ядрами для s-процесса. Это подтверждено наблюдениями спектров звезд-гигантов, принадлежащих шаровым скоплениям. Однако вопрос об эволюции химического состава атмосфер холодных звезд вдоль первой и второй ветвей гигантов остается открытым. Это связано с нашим недостаточным знанием процессов, происходящих во внешних слоях этих звезд. Достаточно сказать, что в спектральном классе К происходит разделение звезд на солнечный и несолнечный типы (имеющих хромосферу и корону или только хромосферу).

Как сказано выше, при анализе содержания химических элементов в атмосферах звезд мы сталкиваемся с ситуацией, когда обнаруживаются звезды с отличием от «стандартного», содержания элементов (например, звезды II типа населения Галактики, звезды типа R CrB, пекулярные, металлические, циркониевые и углеродные звезды и т.д.). Однако подчеркнем, что в тоже время химический состав межзвездного газа в НІІ областях вокруг вновь образующихся звезд и в планетарных туманностях (объектах, которые образовались в поздней стадии эволюции звезды) близок к составу солнечной системы. Более того, содержание химических элементов в других галактиках и квазарах (наиболее старых объектов Вселенной) мало отличается от солнечного. Все это должно быть объяснено теорией образования и эволюции нуклидов. Значительная сложность возникает из-за наличия малого числа стабильных изотопов (островков стабильности, полоса стабильности) среди множества нестабильных изотопов (моря нестабильности) и поэтому кривая распространенности нуклидов в разнообразных объектах Вселенной может видоизменяться в результате процессов радиоактивного распада, которые могут быть различными при тех или иных условиях (вплоть до отсутствия распада). Например, период полураспада ${ }^{99}$ Тс при $\mathrm{T}<10^{8} \mathrm{~K}$ равен, как указывалось ранее $2 \cdot 10^{5}$ лет, а при температуре $\mathrm{T} \sim 3 \cdot 10^{8} \mathrm{~K}$ он составляет пять лет. Образно говоря технеций является не хронометром, а термометром. Особенно это важно учитывать при анализе химического состава метеоритов, при анализе содержания химических элементов в атмосферах звезд, которые можно условно разбить на два типа. Химический состав атмосфер звезд первого типа не загрязнен продуктами звездного ядерного синтеза. У звезд второго типа химический состав их атмосфер может быть разбавлен продуктами термоядерного синтеза и отличаться от химического состава прародительского вещества. Считалось, что звезды диска Галактики (население І-типа) образовались после миллиарда лет жизни нашей Галактики. Однако, в настоящее время найдены звезды диска Галактики с возрастом сравнимым с возрастом шаровых скоплений. При этом необходимо учитывать, что даже у самых старых звезд Галактики, принадлежащих шаровым скоплениям, с металличностью от $-1,5$ до $-3,0 \mathrm{dex}$ отношения в составе тяжелых элементов считались приблизительно в той же пропорции, что и для Солнца. При более тщательном рассмотрении этой проблемы оказывается, что имеются звезды с дефицитом и/или избытком тех или иных нуклидов. «На какой стадии происходит перемешивание межзвездной среды с веществом, прошедшим звездную стадию эволюции?» Известно, это происходит во время вспышек сверхновых І-го и ІІ-го типов (СН I, СН II), возможно во время вспышек новых звезд (Н). Однако звезды, находящиеся на АВГ являются основными поставщиками вновь синтезированных ядер в межзвездную среду в результате истечения вещества (звездный ветер). Массивные, короткоживущие звезды $\left(M>8 M_{\odot}\right)$ синтезируют кислород, элементы $\alpha$-процесса и легкие Z-нечетные и N-четные элементы $(\mathrm{Na}, \mathrm{Al})$. Звезды типа $\mathrm{CH}$ I с $M \sim 1 \div 3 M_{\odot}$ являются источниками элементов группа железа. Эволюция этих звезд происходит медленнее относительно звезд типа СН II. Звезды типа СН I являются основными поставщиками этих элементов в межзвездную среду, т.к. при взрыве происходит полное разрушение звезды. Звезды в стадии АВГ определяют содержание тяжелых нуклидов, синтезированных в результате s-процесса, и нуклиды CNO-группы ( как указывалось выше, значения отношений нуклидов ${ }^{12} \mathrm{C} /{ }^{13} \mathrm{C}$ меняются в атмосферах холодных звезд от 4 до 90). Звезды АВГ имеют вырожденное СО-ядро и два тонких слоевых источника Не и Н, которые включаются попеременно и могут обусловить гелиевые вспышки в 
зависимости от первоначальной массы звезды. Гелиевые (тепловые) вспышки могут способствовать перемешиванию вещества, прошедшего новый термоядерный синтез при горении водорода и горении гелия, с веществом атмосфер звезд. Более того, в этой стадии эволюции возможен вынос на поверхность звезд элементов s-процесса.

Итак, мы имеем, по крайней мере, с четырьмя типами звезд, которые ответственны за распространенность нуклидов в диске Галактики (во Вселенной?). Можно предположить, что их вклад может меняться с возрастом Галактики и при этом можно ожидать лишь частичного перемешивания вещества от вкладов СН II, CH I и АВГ и поэтому звезды данной металличности могут иметь разные распространенности других элементов и их изотопов, в частности, элементов $\alpha$-процесса.

\section{НУКЛИДЫ В МЕТЕОРИТАХ}

Метеориты являются носителями информации о содержании нуклидов в прародительском околосолнечном веществе. Исследования показали на существование в метеоритах дополнительного количества нуклидов, образовавшихся в результате цепочкам радиоактивных распадов. На этом основана хронология метеоритного вещества, как и собственно, хронология земных образцов. Метеориты содержат частицы протосолнечной пылевой материи, которая образовалась истечением из звездных атмосфер в стадии АВГ и во время вспышек сверхновых. Протосолнечными частицами, отношения содержания нуклидов которых не искажены бурными процессами при образовании солнечной системы, в основном являются $\mathrm{SiO}_{2}$ или $\mathrm{SiC}$. Таких частиц, зерен всего 1\% от всей массы метеорита [17]. В целом метеоритная материя характеризуется тремя фазами: силикатной $(74,4 \%)$, троилитной $(5,7 \%)$ и железо-никелевой $(19,6 \%)$. Эти данные получены из анализа обычных хондритов, которые являются наиболее распространенными метеоритами. Метеориты чрезвычайно негомогенны. Индивидуальные пробы метеоритов могут иметь разный состав изотопов $\mathrm{Al}, \mathrm{O}, \mathrm{Si}$ и С. Это отражает неоднородность содержания нуклидов в Галактике даже в околосолнечном пространстве. Многие пробы содержат разные отношения нуклидов инертных газов и элементов $\mathrm{Ba}, \mathrm{Nd}, \mathrm{Sm}$ и Dy. Исследования показали, что даже в одном SiC образце содержатся разные содержания нуклидов $\mathrm{Sr}, \mathrm{Zr}$ и Мо. Таким образом, метеориты дают важную информацию о звездной эволюции нуклидов. Отметим, что содержания нуклидов в разных образцах метеоритов значительно отличаются от содержания их в солнечной системе. Следовательно, эти пробы представляют собой реальное звездное вещество и оно сохранило прародительское отношение нуклидов. Поэтому протосолнечные частицы дают информацию о реальных источниках звездной эволюции нуклидов и реальном эволюционном статусе звезды (холодный гигант или гигант асимптотической ветви). Типичными представителями первичной солнечной материи являются углистые хондриты, которые содержат наибольшее количество летучих элементов. Все остальные метеориты возникли в результате дифференциации этого прародительского вещества. Вариации отношения ${ }^{16} \mathrm{O} /{ }^{17} \mathrm{O}$ отражает различия звезд по массе, а вариации отношения ${ }^{16} \mathrm{O} /{ }^{18} \mathrm{O}$ могут быть объяснены только первоначальным различием этого отношения в протозвездном веществе. Наличие избытка ${ }^{26} \mathrm{Mg}$ может быть объяснено радиоактивным распадом ${ }^{26} \mathrm{Al}$. Нуклид ${ }^{26} \mathrm{Al}$ образуется при высокой температуре в слоевом источнике горения Н и, следовательно, он может образоваться только на эволюционной стадии AGB. Часть вещества метеоритов образовалась во время вспышек сверхновых. $\mathrm{SiC}$ в метеоритах образуются в углеродных звездах. Состав тяжелых нуклидов обусловлен s-процессом ( $\mathrm{Kr}, \mathrm{Sr}, \mathrm{Zr}, \mathrm{Mo}, \mathrm{Xe}, \mathrm{Ba}, \mathrm{Nd}, \mathrm{Sm}$ and Dy).

Более того, анализ химического состава метеоритов со всей определенностью показал, что солнечная система в момент образования также была неоднородной. У некоторых метеоритов обнаружены аномалии в изотопном составе $\mathrm{O}, \mathrm{Mg}, \mathrm{Ca}, \mathrm{Ti}, \mathrm{Sr}, \mathrm{Ba}, \mathrm{Nd}$ и $\mathrm{Sm}$, а также аномалии в изотопном содержании редких на Земле, но не во Вселенной, нуклидов $\mathrm{Ne}, \mathrm{Kr}$ и Хе. Более того, пыль в непосредственной окрестности Солнца после выметания газа, по всей вероятности, была нагрета до температуры $2000 \mathrm{~K}$ солнечным излучением. Это привело к ее испарению и новой конденсации. При этом произошло взаимодействие с другими областями солнечной системы, что привело к изменениям в содержании химических элементов прародительского вещества. 


\section{ЗАКЛЮЧЕНИЕ}

В последнее время наметился существенный успех в интерпретации кривой распространенности нуклидов. Можно сделать основной вывод, что основными термодинамическими котлами, где варятся нуклиды, являются звезды. При этом разные нуклиды образуются в недрах звезд, которые находятся на разной стадии эволюции - протозвезд, звезд-карликов, звезд-гигантов и сверхгигантов, звезд асимптотической ветви гигантов, сверхновых I и II типов, белых карликов и нейтронных звезд. Необходимо заметить, что в недрах звезд кроме основных ядерных реакций могут происходить и другие реакции. Это подтверждается содержанием определенных элементов, например Na. Содержание $\mathrm{Na}$ в атмосферах звезд-гигантов и сверхгигантов говорит, что в недрах звезд-карликов происходит неоно-натриевый цикл ${ }^{22} \mathrm{Ne}(\mathrm{n}, \gamma){ }^{23} \mathrm{Na}$ и вынос натрия на стадии ГП или при выходе на ВГ. В подтверждение вышесказанного можно привести еще один пример. Отношение ${ }^{16} \mathrm{O} /{ }^{18} \mathrm{O}$ зависит от той или иной остановки последовательности ядерных реакций горения азота ${ }^{14} \mathrm{~N}(\alpha, \gamma){ }^{18} \mathrm{~F}\left(\beta^{+}, v\right)^{18} \mathrm{O}(\alpha, \gamma){ }^{22} \mathrm{Ne}(\alpha, n)^{25} \mathrm{Mg}$ на стадии АВГ. Останавливается ли эта цепочка реакций на нуклиде ${ }^{18} \mathrm{O}$ или происходят ядерные реакции до ${ }^{22} \mathrm{Ne}$, или до ${ }^{25} \mathrm{Mg}$. При температурах характерных для горения гелия в результате тепловых вспышек скорость реакции ${ }^{18} \mathrm{O}(\alpha, \gamma)^{22} \mathrm{Ne}$ очень высока и поэтому содержание нуклида ${ }^{18} \mathrm{O}$ практически не меняется. В тоже время должно меняться содержание нуклида ${ }^{22} \mathrm{Ne}$ и этот теоретический прогноз находит подтверждение в большом содержании неона в планетарных туманностях.

В кратком обзоре мы не останавливались на такой важной проблеме, как эволюция нуклидов при вспышках сверхновых и новых. Это связано с тем, что теория образования элементов в результате взрыва гетерогенной (слоистой) предсверхновой звезды еще недостаточно разработана, хотя и имеются определенные успехи в этой области. На поздней стадии эволюции звезды образуются слоевые источники, которые окружают неон-кремневое или железное ядро. Масса слоевых источников и состав нуклидов определяется общей массой звезды и темпами потери массы. Не разработана теория эволюции звезд с учетом потери массы. Открытым остается вопрос точного учета конвекции. Несмотря на эти трудности был проведен расчет эволюции массивной звезды на поздней стадии. Во любом случае взрыв массивной сверхновой (СН II) не производит нуклиды тяжелее кремния. Поэтому более важную роль в ядерном синтезе должны играть сверхновые малых масс (СН I). Именно они являются поставщиками в межзвездную среду элементов железного пика $(50<A<65)$. В последнее время выявлена зависимость $\mathrm{r}$-процесса от массы и радиуса протонейтронной звезды. Для объяснения происхождения тяжелых нуклидов разработан процесс, который условно называется r-процессом с обратной связью или rbc-процесс. С помощью этого процесса стала возможным решение ряда принципиальных проблем, которые возникали при использовании обычного r-процесса. Основная проблема - это источник свободных нейтронов. Для детального ознакомления с этой проблемой можно обратиться к монографии Бисноватого-Когана [1].

1.Бисноватый-Коган Г.С. Физические вопросы теории звездной эволюции. - М.: Наука, 1989. - 481 с.

2.Боярчук А.А., Антипова Л.И., Боярчук М.Е., Саванов И.С. Сравнительный анализ содержания химических элементов в атмосферах красных гигантовразных возрастных групп // Астрон. Ж. - 2001. - 78. - С.349-358.

3.Гопка В.Ф., Панчук В.Е., Комаров Н.С. Содержание скандия, титана и элементов группы железа в атмосферах $\gamma$ Стрелы и $\alpha$ Тельца. //Астрофиз. исслед. (Изв.САО). - 1989. - 27. - С.13-17.

4.Гопка В.Ф., Комаров Н.С., Мишенина Т.В., Ющенко А.В. Содержание элементов r-, s-процессов в атмосферах Кгигантов // Письма в Астрон. Ж. - 17. - С.368-374.

5.Комаров Н.С. Холодные звезды-гиганты. - Одесса: Астропринт, 1999. - 213 с.

6. Физический энциклопедический словарь / Изд-во Советская энциклопедия. - 1966 - т.5 - С.186-191.

7.Anders E., Grevesse N. Abundances of the Elements: Meteoritic and Solar//Geoch. et Cosmoch.Acta. - 1989. - 53. P.197-203.

8.Burbidge E.M., Burbidge G.R., Fowler W.A., Houle F. Synthesis of the elements in stars // Rev.Mod.Phys. - 1957 - 29. P.547-650.

9. Gray G.M., Compston W. Excess ${ }^{26} \mathrm{Mg}$ in the Allende meteorites // Nature. - 1974. - 251. - P.495-497.

10. Grevesse N., Sauval J. Standard Solar Composition // Space Science Review.-1998. - 85. - P.161-174. 
11. Harris M.J., Lambert D.L. The ${ }^{12} \mathrm{C} /{ }^{13} \mathrm{C}$ and ${ }^{16} \mathrm{O} /{ }^{18} \mathrm{O}$ rations in the solar photosphere // Month.Not.Roy.Astron.Soc. 1987. - 224. - P.237-255.

12. Harris M.J., Lambert D.L., Smith V.V. Oxygen isotopic abundances in evolved stars. // Astrophys.J. - 1988. - 325. P.768-775.

13. Merril P.W. Spectroscopic observations of stars of class S. // Astrophys.J. - 1952. - 116. - P.21-26.

14. Lallansio J.C., Malaney N.A. Photofission production of technetium and synthetic asymptotic giant branch evolution // Astrophys.J. - 1989. - 347. - P.989-997.

15. Smith V.V., Lambert D.L. S-process-enriched cool stars with and without technetium: clues to asymptotic giant branch and binary star evolution // Astrophys.J. -1988. - 333. - P.219-226.

16. Tomkin J., Lambert D.L. Magnesium isotopes and s-process elements in the barium star HR774. / Astrophys.J. - 1979. 227. - P.209-219.

17. Zinner E., Amari S. Asymptotic giant branch stars / IAU Symposium № 191. - 1999. -P.59-68.

Поступила в редакцию 30.08 .2001 